\title{
EFFETS DU SOJA ET DES PHYTO-OESTROGÈNES SUR LA VITELLOGÉNÈSE ET L'ENDOCRINOLOGIE STÉROÏDIENNE DE LA TRUITE ARC-EN-CIEL ET DE L'ESTURGEON SIBÉRIEN. APPROCHES IN VIVO ET IN VITRO.
}

\author{
C. BENNETAU-PELISSERO (1, 2), S. KAUSHIK (3), J. SUMPTER (4), A. FOSTIER (5), \\ F. LE GAC (5), Y. VALOTAIRE (6), B. DAVAIL-CUISSET (2) et F. LE MENN (2)
}

(1) ENITA de Bordeaux, 1 cours du Général de Gaulle, B.P. 201, 33175 Gradignan Cedex, France.

(2) Laboratoire de Biologie de la Reproduction des Poissons, Avenue des Facultés, 33405 Talence Cedex, France.

(3) Laboratoire mixte INRA-IFREMER de Nutrition des Poissons, 64310 St-Pée-sur-Nivelle, France.

(4) Department of Biology and Biochemistry, Brunel University, Uxbridge. Middlesex, UB8 $3 \mathrm{PH}$, United Kingdom.

(5) Laboratoire INRA de Physiologie des Poissons, Campus de Beaulieu, Avenue du Général Leclerc, 35042 Rennes, France.

(6) Laboratoire Université CNRS d'Endocrinologie Moléculaire de la Reproduction, Université de Rennes I, Campus de Beaulieu, Avenue du Général Leclerc, 35042 Rennes, France.

\section{RÉSUMÉ}

La mise au point d'un test de sexage des esturgeons sibériens, basé sur la détection de la vitellogénine (VTG) plasmatique, a permis de mettre en évidence cette protéine complexe oestrogéno-dépendante dans le plasma des poissons femelles, mâles et immatures de cette espèce. L'hypothèse d'une contamination oestrogénique d'origine environnementale a été confirmée par un test basé sur l'administration de différents aliments. Chez les témoins sans oestrogènes, la VTG disparaissait en quelques semaines du plasma des poissons. Avec un aliment du commerce ou un aliment à base de soja, les taux de VTG augmentaient de manière significative. Plus tard, un effet oestrogénique du soja administré par voie orale, quoique faible, a été mis en évidence in vivo chez la truite.

Le soja est connu pour contenir des phyto-oestrogènes qui ont montré leurs effets oestrogéniques in vivo par injection chez de jeunes esturgeons. Ils ont ensuite été testés chez des truites arc-en-ciel in vitro en culture d'hépatocytes. Les résultats diffèrent suivant que l'on s'adresse à des femelles en début de vitellogénèse d'une part ou à de très jeunes poissons et des mâles d'autre part. Cette différence est provisoirement expliquée par des différences entre les deux sexes et entre poissons adultes et très jeunes dans les niveaux de récepteurs de l'oestradiol hépatiques disponibles. 
In vitro chez la truite, un effet inhibiteur d'un des phyto-oestrogènes majeurs, l'équol, a été montré sur l'activité aromatase ovarienne. Ce composé se lie in vitro aux protéines porteuses de stéroïdes (SBP) de truite alors que chez l'esturgeon, ce sont deux phytooestrogènes - l'équol et la génistéine - qui entrent en compétition avec la testostérone pour les sites de liaison de la SBP. Les tests in vivo réalisés chez la truite montrent que tous les lots de matières premières ne semblent pas équivalents en termes de concentrations de phyto-oestrogènes et un dosage de ces composés nous paraît essentiel à mettre au point pour pouvoir corréler les doses et les effets observés.

\title{
SOY AND PHYTOESTROGENS. THEIR EFFECTS ON VITELLOGENESIS AND STEROID ENDOCRINOLOGY IN THE RAINBOW TROUT AND IN THE SIBERIAN STURGEON. IN VIVO AND IN VITRO APPROACHES.
}

\begin{abstract}
The development of a new sex determination test of Siberian sturgeon based on vitellogenin (VTG) detection in plasma led to identify this complex factor in plasma from male, female and immature fish of this species. The hypothesis of an estrogenic contamination from an environmental origin was confirmed by a test based on the feeding of fish on different diets. In control fish fed with a casein-based diet free of any estrogenic compound, VTG disappeared from the plasma within 15 weeks. With a fish meal-based diet as well as with a soy-based diet, the plasma VTG levels raised significantly. Later, an estrogenic effect of soy orally administrated was demonstrated in vivo in the rainbow trout although it is lower than that observed in sturgeon.
\end{abstract}

Soya is known to contain phytoestrogens which were demonstrated to be estrogenic when injected in vivo in yearling Siberian sturgeon. These compounds were then tested in vitro in hepatocyte culture obtained from rainbow trout. Results were shown to be different between male and female at the onset of vitellogenesis. This difference is explained, up to now, by a difference in the initial levels of estradiol receptor available between males and females and between very young fish and fish undergoing their gametogenesis.

In vitro, in the rainbow trout equol, one of the major phytoestrogens was found to have an inhibitory effect on the ovarian aromatase activity. This compound can also bind to the rainbow trout Steroid Binding Protein (SBP) whereas in sturgeon at least two compounds namely equol and genistein can compete for SBP with testosterone.

In vivo tests realised in the rainbow trout recently showed that all vegetable crude matter are not equivalent in terms of phytoestrogens contents and it seems to us crucial to set up a new assay of these compounds to be able to correlate doses in diets and effects recorded in fish.

\section{INTRODUCTION}

Les phyto-oestrogènes sont des oestrogènes de l'environnement que l'on rencontre naturellement dans de nombreux végétaux dont certains entrent couramment dans la composition des aliments destinés aux animaux d'élevage ou à l'homme (SETCHELL, 1985). Ces phyto-oestrogènes peuvent être répartis en plusieurs familles chimiques dont les plus importantes sont les isoflavones, les lignanes, les lactones d'acides résorciliques et les coumestanes (SETCHELL, 1985). Les isoflavones, telles la biochanine A, la daidzéine, la formononétine et la génistéine, sont surtout présentes dans les légumineuses 
(FARNWORTH et al., 1975). Parmi ces végétaux, ceux chez qui les teneurs sont les plus élevées sont le soja et les trèfles (STOB, 1983). Pourtant, on en trouve également dans le lupin, les pois fourragers ou dans des fruits comme les cerises ou les oranges. La famille des coumestanes est surtout représentée par le coumestrol qui peut atteindre des concentrations très élevées dans la luzerne (SHERWOOD et al., 1970). Les lignanes, notamment l'entérodiol et l'entérolactone, sont apportés via certains précurseurs chimiques par les graines (lin, sésame...) (AXELSON et al., 1982). Les lactones d'acides résorciliques regroupent notamment deux mycotoxines fortement oestrogéniques, la zearalénone et le zéralénol, qui apparaissent sur des stocks de blé ou de maïs après infestation par des micro-organismes (HESSELTINE et al., 1978).

Un regain d'intérêt se fait jour actuellement autour des isoflavones notamment depuis que l'une d'entre elles, la génistéine, s'est montrée un inhibiteur spécifique des tyrosines kinases (THOMPSON, 1993). Ces enzymes qui interviennent à une place prépondérante dans les relations intercellulaires faisant intervenir des facteurs de croissance et/ou des médiateurs humoraux sont entre autres impliquées dans les phénomènes de tumorisation. On explique notamment aujourd'hui par cette propriété l'action anticancéreuse de certains régimes alimentaires végétariens basés sur une forte ingestion de soja (BARNES, 1995). Bien que les mécanismes de prévention contre le cancer soient de plus en plus étudiés notamment grâce aux fonds privés des industries productrices et transformatrices de soja, il n'en reste pas moins que la première activité que l'on ait historiquement attribuée à ces composés est une potentialité oestrogénique et que celle-ci demeure.

A l'heure où la raréfaction des stocks de poissons sauvages fait suite à la surpêche et compromet entre autres l'obtention de farines de poisson, à l'heure où l'interêt en aquaculture se porte aussi vers une meilleure gestion des déchets, les industriels de l'alimentation animale tendent à remplacer dans leurs produits leur source de protéine animale par des sources de protéines végétales. Parmi ces produits de substitution le soja tient une place prépondérante. II nous semble dès lors légitime de nous intéresser à l'activité des produits susceptibles d'être apportés par ces matières premières d'origine végétale. Dans la mesure où leurs effets oestrogéniques ont été rapportés chez les mammifères, nous les avons étudiés chez deux modèles de poissons chronologiquement l'esturgeon puis la truite. La littérature s'étoffant ces dernières années d'un grand nombre de travaux, nous avons aussi cherché à connaître les effets de ces molécules sur certaines étapes du transfert de l'information oestrogénique. Un ensemble d'informations peut aujourd'hui être dégagé qui fait apparaître avant tout la variabilité interspécifique des poissons face à cette contamination oestrogénique et, par ailleurs, l'importance de la présence de récepteurs de l'oestradiol pour permettre ces effets.

\section{LES EFFETS OESTROGÉNIQUES IN VIVO}

\section{Chez l'esturgeon}

C'est sur cette espèce qu'ont été montrés les premiers effets des phyto-oestrogènes (PELISSERO et al., 1990a). A la suite de travaux visant à identifier la vitellogénine (VTG) dans le plasma des esturgeons sibériens Acipenser baeri, nous nous sommes rendus compte que cette molécule complexe précurseur des réserves vitellines de l'oeuf et strictement oestrogéno-dépendante était présente dans le plasma de tous les animaux en élevage de plus d'un an (PELISSERO et al., 1989a) (Figure 1).

La gamétogénèse femelle commençant tardivement chez cette espèce, précisément à partir de quatre ans, nous avons dès lors invoqué une contamination oestrogénique. Plusieurs sources de contamination pouvaient être retenues mais un faisceau de présomptions nous a amené à considérer de manière plus particulière la source alimentaire. 
Taux plasmatiques de VTG chez l'esturgeon sibérien élevé en pisciculture

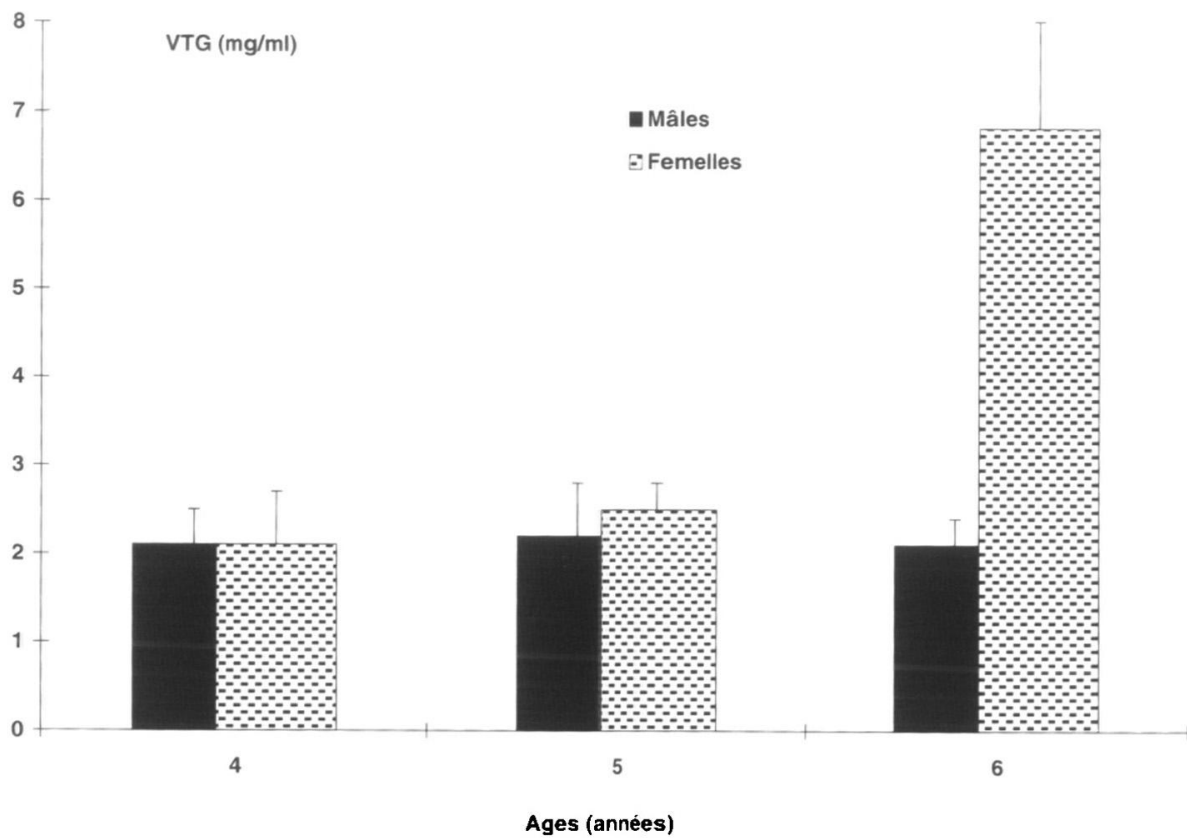

Figure 1

Taux plasmatiques de vitellogénine enregistrés chez des esturgeons mâles, femelles et immatures, élevés en pisciculture.

\section{Figure 1 \\ Vitellogenin plasma levels recorded in male, female and immature Siberian sturgeon reared in fish farm.}

Un essai consistant à comparer l'effet oestrogénique de différents aliments obtenus à partir de bases protéiques différentes a été mené (PELISSERO et al., 1990b). Trois aliments ont été testés. L'un était basé sur une farine de poisson mais sa composition exacte nous restait inconnue car protégée par le sceau du secret industriel, un autre aliment contenait essentiellement de la caséine et était dépourvu de tout composé oestrogénique. II constituait notre aliment témoin. Enfin, le dernier aliment contenait $40 \%$ de soja. La composition des aliments testés est donnée par le Tableau I.

Les niveaux initiaux de VTG chez les animaux âgés de 1,5 an à leur arrivée au laboratoire n'étaient pas nuls, ce qui laissait pressentir la contamination oestrogénique en élevage que nous souhaitions mettre en évidence. Les résultats, obtenus en termes de VTG après 15 semaines d'administration des différents régimes et suivant un rythme de prélèvement sanguin toutes les trois semaines, sont présentés dans la Figure 2.

On observe que l'aliment témoin à base de caséine provoque la disparition de la VTG dans le plasma des animaux. Ceci montre qu'en l'absence de contamination oestrogénique, les esturgeons immatures choisis pour cette expérience n'auraient pas dû présenter de VTG dans leur plasma. Les animaux ayant reçu l'aliment commercial à base de farine de poisson ont vu leurs taux plasmatiques de VTG augmenter de $0,6 \mathrm{mg} / \mathrm{ml}$, valeur initiale à plus de $1,6 \mathrm{mg} / \mathrm{ml}$ après 15 semaines d'alimentation. Ceci, par comparaison au témoin, démontrait le caractère oestrogénique de l'aliment commercial chez l'esturgeon. Enfin, chez les animaux nourris avec l'aliment à base de soja les niveaux moyens de VTG ont été multipliés par 10, passant dans le même temps de 0,6 à plus de $6 \mathrm{mg} / \mathrm{ml}$. Si l'on compare donc chez l'esturgeon les effets oestrogéniques d'un aliment à base de farine de poisson et ceux d'un aliment à base de soja, on est en droit de considérer la contamination due au soja comme étant beaucoup plus problématique. 


\section{Tableau I}

Composition des trois aliments testés sur les esturgeons sibériens.

\section{Table I}

Composition of the diets tested on the Siberian sturgeon.

\begin{tabular}{lccc}
\hline Ingrédients & $\begin{array}{c}\mathbf{S C} \\
(\mathbf{g} / \mathbf{1 0 0} \mathbf{g})\end{array}$ & $\begin{array}{c}\mathbf{S S} \\
(\mathbf{g} / \mathbf{1 0 0} \mathbf{~ g})\end{array}$ & T$^{\mathbf{a}}$ \\
\hline Farine de soja & - & 30 & \\
Caséine (vit free) & 45 & 30 & \\
Amidon gélatinisé & 20 & 20 & \\
Amidon cru & 17 & 3 & \\
Huile de poisson & 10 & 9 & \\
Prémélange vitaminé & 2 & 2 & \\
Prémélange minéral & 5 & 5 & 11,8 \\
Alginate de sodium & 1 & 1 & 50,5 \\
Humidité (\%) & 14,5 & 17,2 & 9,3 \\
Protéines (\% de matière sèche) & 45 & 45,1 & 20,0 \\
Lipides (\% de matière sèche) & 9,7 & 9,8 & \\
Energie (kJ/g de matière sèche) & 22,0 & 21,4 & \\
Cendres (\% de matière sèche) & 5,5 & 7,1 & \\
\hline
\end{tabular}

Note. La liste des ingrédients est complète pour les deux régimes synthétiques expérimentaux (SC et SS), mais pas pour l'aliment commercial (T) à cause du secret commercial.

a L'aliment commercial. La composition de cet aliment n'est pas dévoilée mais on sait qu'il contient des farines de poisson ainsi que des concentrés protéiques animaux et végétaux.

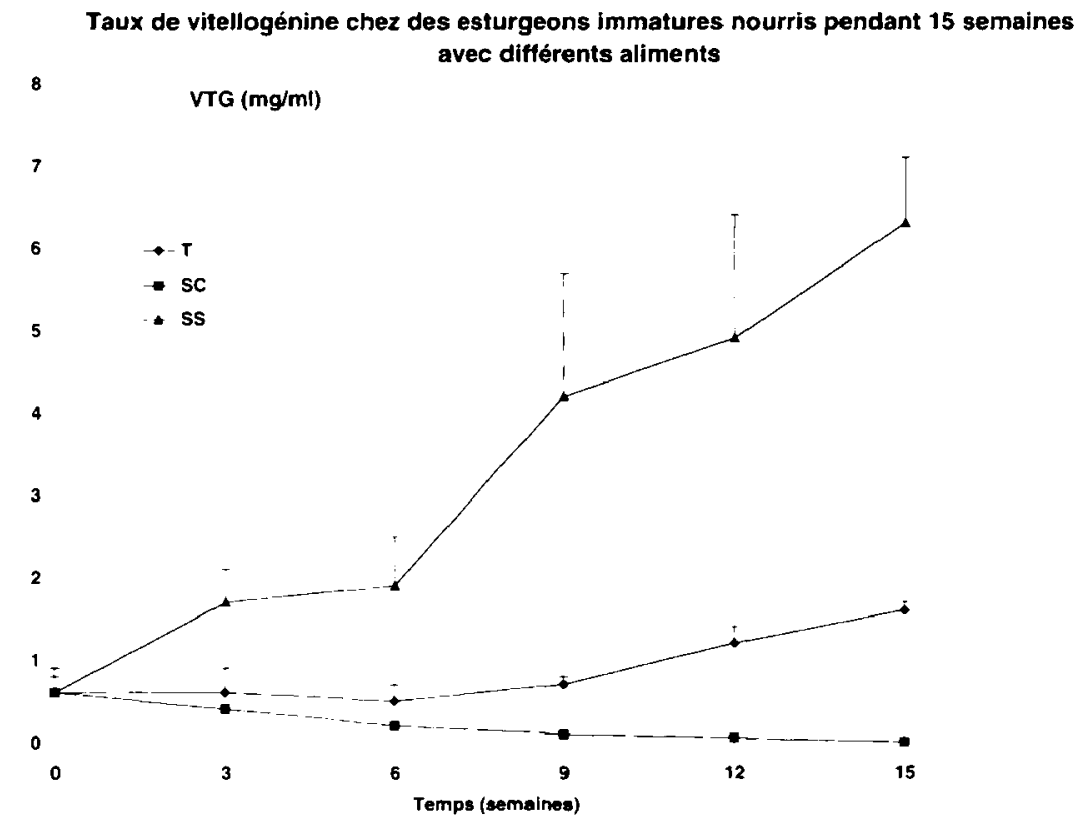

Figure 2

Evolution des taux de VTG plasmatiques chez des esturgeons sibériens de 1 an et demi (immatures) nourris avec différents aliments ( $T$ : aliment pour truite du commerce ; SS : aliment à base de soja ; SC : aliment à base de caséine).

Figure 2

Vitellogenin plasma levels in immature Siberian sturgeon (one year and a half old) fed on various diets (T : commercial trout diet ; SS : soya bean-based diet ; SC : casein-based diet). 
Il est certain que ce phénomène a été mis en lumière grâce aux particularités spécifiques de l'esturgeon sibérien dont nous commençons aujourd'hui à cerner certains éléments mais qui ne seraient sans doute pas apparus aussi nettement chez une autre espèce. L'objet de cet article est de donner, outre les faits, des débuts d'interprétations des mécanismes mis en oeuvre.

Après l'obtention de ces résultats, nous avons cherché à tester les effets oestrogéniques des composés isoflavoniques connus dans le soja. Pour ce faire, les composés purs ont été synthétisés et injectés à de très jeunes esturgeons de 4 mois nourris une semaine avant le début de l'expérience et ensuite pendant toute sa durée avec des asticots. Compte tenu des doses nécessaires pour chaque composé et des difficultés de synthèse, une seule dose a été testée pour chaque composé en s'inspirant de l'effet de ces composés chez les mammifères. Les taux de VTG mesurés chez les témoins et chez les animaux injectés avec les différents produits sont présentés dans le Tableau II.

\section{Tableau II}

Taux plasmatiques de vitellogénine induits par injection intrapéritonéale de phytooestrogènes chez de jeunes esturgeons ( $20 \mathrm{~g}$ de poids moyen).

\section{Table II}

Vitellogenin plasma levels induced by intra-peritoneal injection of phytoestrogens in yearling Siberian sturgeon ( $20 \mathrm{~g}$ mean weight).

\begin{tabular}{lccc}
\hline $\begin{array}{l}\text { Composés } \\
\text { OEstrogéniques }\end{array}$ & $\begin{array}{c}\text { Doses } \\
(\mathbf{m g} / \mathbf{m l})\end{array}$ & $\begin{array}{c}\text { Vitellogénine } \\
(\mu \mathbf{g} / \mathbf{m l})\end{array}$ & Nb de poissons \\
\hline Témoin & - & $<0,1$ & $(\mathrm{n}=5)$ \\
Formononétine & 0,5 & $<0,1$ & $(\mathrm{n}=4)$ \\
Daidzéine & 0,2 & $2,00 \pm 0,06$ & $(\mathrm{n}=4)$ \\
Equol & 0,05 & $8,8 \pm 2,8$ & $(\mathrm{n}=3)$ \\
Biochanine A & 0,5 & $98 \pm 14$ & $(\mathrm{n}=4)$ \\
Génistéine & 0,2 & $213 \pm 56$ & $(\mathrm{n}=4)$ \\
Coumestrol & 0,05 & $272 \pm 98$ & $(\mathrm{n}=4)$ \\
OEstradiol 17 $\beta$ & 0,0001 & $246 \pm 97$ & $(\mathrm{n}=4)$ \\
\hline
\end{tabular}

On observe que l'oestrogénicité des composés telle qu'elle est rapportée chez les mammifères n'est pas exactement similaire à celle trouvée chez l'esturgeon. D'après la littérature, chez les mammifères, l'échelle d'oestrogénicité est la suivante :

coumestrol $=$ équol $>$ génistéine $>$ biochanine $A$ > daidzéine $>$ formononétine .

Chez l'esturgeon in vivo on aurait plutôt, compte tenu des différentes doses testées :

coumestrol > génistéine > biochanine $A \cong$ équol > daidzéine (la formononétine n'étant pas ou que très peu active).

Rappelons ici que les animaux choisis étaient très jeunes et ne possédaient sans doute que de très faibles taux de récepteurs hépatiques des oestrogènes.

\section{Chez la truite arc-en-ciel}

Récemment, une expérience comparant comme pour l'esturgeon les effets oestrogéniques de différents aliments basés sur des concentrés protéiques différents a été réalisée chez la truite (PELISSERO-BENNETAU et al., 1997). Dans cette expérience, on a 
comparé les effets oestrogéniques d'un aliment à base de farine de poisson constituant notre témoin à trois aliments à base de concentrés protéiques végétaux. Parmi les aliments testés, deux étaient préparés à partir de concentrés protéiques de soja d'origines différentes et un à base de gluten de maïs. La composition des aliments utilisés dans ce test est donnée dans le Tableau III. Les poissons pesaient $70 \mathrm{~g}$ au début de l'expérience et les régimes ont été distribués pendant 12 semaines. A la fin de l'expérience, les poids moyens mesurés sur les animaux étaient significativement plus faibles chez les animaux ayant reçu les aliments TE (concentré protéique de soja $n^{\circ} 2$ ) et TG (aliment à base de gluten de maïs). Les taux de VTG mesurés chez les femelles d'une part et chez les mâles d'autre part sont rapportés dans la Figure 3.

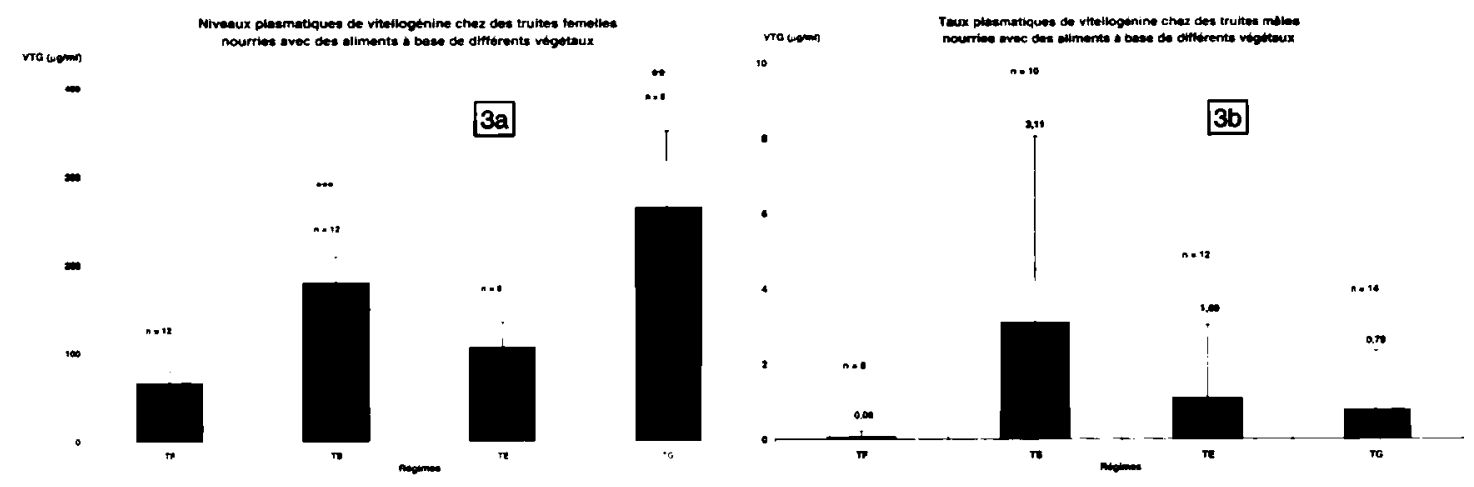

Figure 3a

Niveaux de vitellogénine enregistrés chez de jeunes femelles en début de vitellogénèse après 12 semaines d'alimentation avec des régimes à base de protéines végétales (TF : aliment témoin à base de farine de poisson ; TS et TE : à base de concentrés protéiques de soja ; TG : à base de gluten de maïs).

\section{Figure 3a}

Vitellogenin plasma levels measured in young female rainbow trout at the beginning of vitellogenesis fed for 12 weeks on various vegetable-based diets. (TF : control diet based on fish meal ; TS and TE : soya bean-based diet ; TG : corn gluten-based diet).

\section{Figure 3b}

Niveaux de vitellogénine enregistrés chez de jeunes mâles en début de spermiogénèse après 12 semaines d'alimentation avec des régimes à base de protéines végétales (TF : aliment témoin à base de farine de poisson ; TS et TE : à base de concentrés protéiques de soja ; TG : à base de gluten de maïs).

\section{Figure 3b}

Vitellogenin plasma levels measured in young males at the beginning of spermatogenesis fed for 12 weeks on various vegetable-based diets. (TF : control diet based on fish meal ; TS and TE : soya bean-based diet ; TG : corn gluten-based diet).

On observe qu'une induction significative est observée avec l'aliment TS (basé sur le concentré de protéines de soja $n^{\circ} 1$ ) et avec l'aliment TG (à base de gluten de maïs). La mesure des taux de génistéine et de daidzéine réalisée dans ces aliments n'explique pas les différences de réactivité observée chez les poissons. En effet, les taux de génistéine et de daidzéine sont plus élevés dans l'aliment TE que dans l'aliment TS. Ceci indique que ni la génistéine ni la daidzéine ne sont les composés responsables dans ce cas de l'activité oestrogénique de l'aliment. D'autres molécules comme des herbicides, des pesticides ou encore les phyto-oestrogènes zearalénol et coumestrol, pourraient être à l'origine de l'effet observé avec les aliments TS et TG. Chez les mâles en revanche, l'effet est masqué par une 
très forte hétérogénéité dans la réponse qui rend tous les résultats non significatifs. Notons ici que dans le cas des aliments préparés à partir de protéines végétales, les mâles pouvaient toujours être séparés en 2 groupes, $20 \%$ d'entre eux environ semblaient réagir et présentaient des taux de VTG non nuls alors que $80 \%$ des autres ne présentaient pas de VTG dans leur plasma, au moins à la sensibilité de notre dosage.

Là encore, comme chez les petits esturgeons de quelques mois, il semble que l'on puisse attribuer le manque d'action des phyto-oestrogènes à un faible taux initial des récepteurs des oestrogènes hépatiques. Ceci devra pourtant être confirmé notamment par des analyses de liaison sur des extraits cellulaires.

\section{LES EFFETS OESTROGÉNIQUES IN VITRO}

II est assez aisé chez la truite de tester in vitro les capacités oestrogéniques de diverses substances en travaillant sur des cultures primaires d'hépatocytes (PELISSERO et al., 1991 ; 1993). Les phyto-oestrogènes ont d'abord été testés en système simple dans lesquels c'était la VTG elle-même qui était dosée dans les milieux de culture après 12 jours de culture. Sur des cultures d'hépatocytes obtenues à partir de poissons femelles, nous avons montré plusieurs choses.
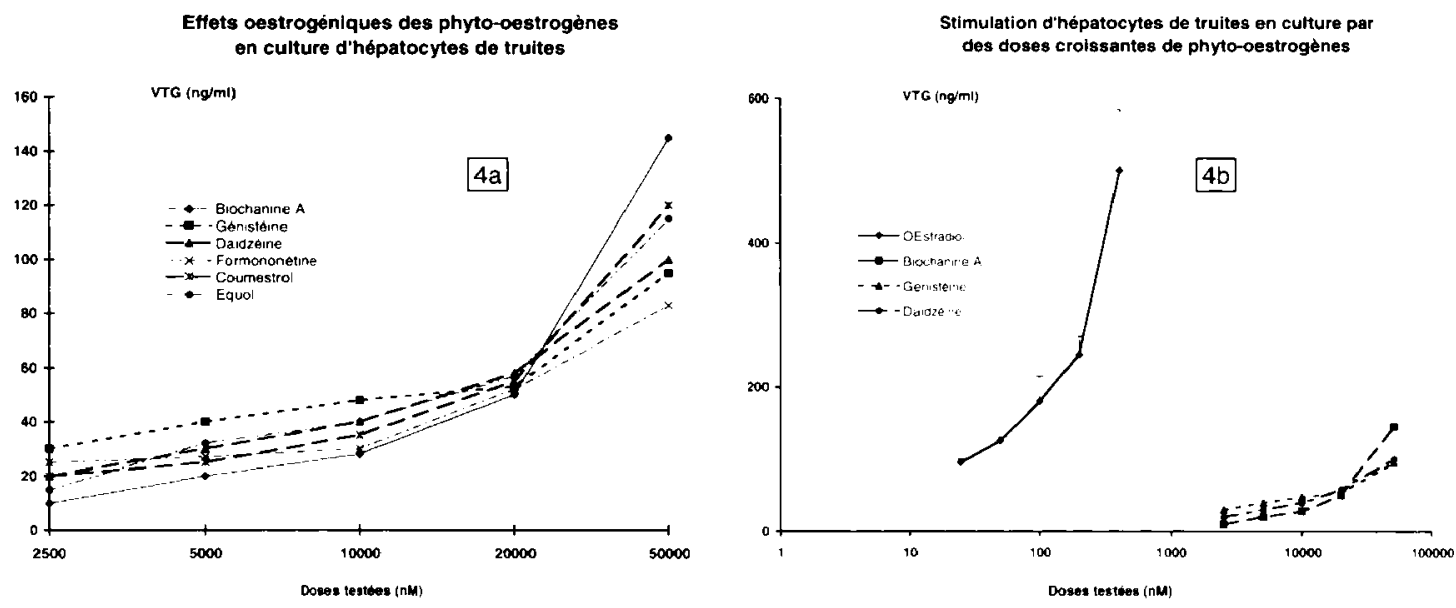

Figure 4a

Effets oestrogéniques relatifs des phyto-oestrogènes en culture d'hépatocytes.

\section{Figure 4a}

Relative estrogenic potencies of phytoestrogens in hepatocyte culture.

\section{Figure 4b}

Effets oestrogéniques de trois phyto-oestrogènes en culture par rapport à celui de l'oestradiol.

\section{Figure 4b}

Estrogenic effects of three phytoestrogens compared to estradiol.

En premier lieu, l'oestrogénicité des phyto-oestrogènes est non statistiquement différente d'un composé à l'autre (Figure 4a), ce qui entre en opposition avec les résultats précédemment établis in vivo chez l'esturgeon. Par ailleurs, leurs potentialités oestrogéniques 
sont de l'ordre de 1000 à 2000 fois moindre que celles de l'oestradiol (Figure 4b) alors que chez les mammifères elles sont comprises entre 1000 (pour le coumestrol et l'équol) et 10000 pour la biochanine $A$ et la formononétine. On voit par là que l'activité oestrogénique des phyto-oestrogènes, même au niveau d'un système simplifié comme l'est la culture d'hépatocytes, est déjà compliquée par une dégradation possible des composés par les enzymes du compartiment hépatique. Enfin, en testant l'effet du tamoxifène inhibiteur spécifique de la liaison des oestrogènes au récepteur de l'oestradiol, nous avons confirmé ce qui était déjà admis chez les mammifères, à savoir que l'effet oestrogénique des phyto-oestrogènes passait bien par une liaison au récepteur de l'oestradiol.

L'homogénéité des potentialités oestrogéniques, obtenues en culture d'hépatocytes pour les différents phyto-oestrogènes, a précédemment été expliquée par une métabolisation des composés dans les hépatocytes dotés d'activités enzymatiques multiples qui auraient ainsi transformé tous les phyto-oestrogènes en un dérivé très oestrogénique commun. Pourtant, des analyses des sucs biliaires de truites exposés à des phyto-oestrogènes ne montrent pas l'apparition d'un tel composé (CRAVEDI, communication personnelle). Faut-il pour autant exclure une transformation et une action locale de ces composés? Par ailleurs, rappelons ici à toutes fins utiles que les tests avaient été réalisés sur des hépatocytes prélevés chez de jeunes femelles en début de vitellogénèse. Chez ces dernières, les taux du récepteur de l'oestradiol sont déjà assez importants pour mettre en évidence une activité oestrogénique.

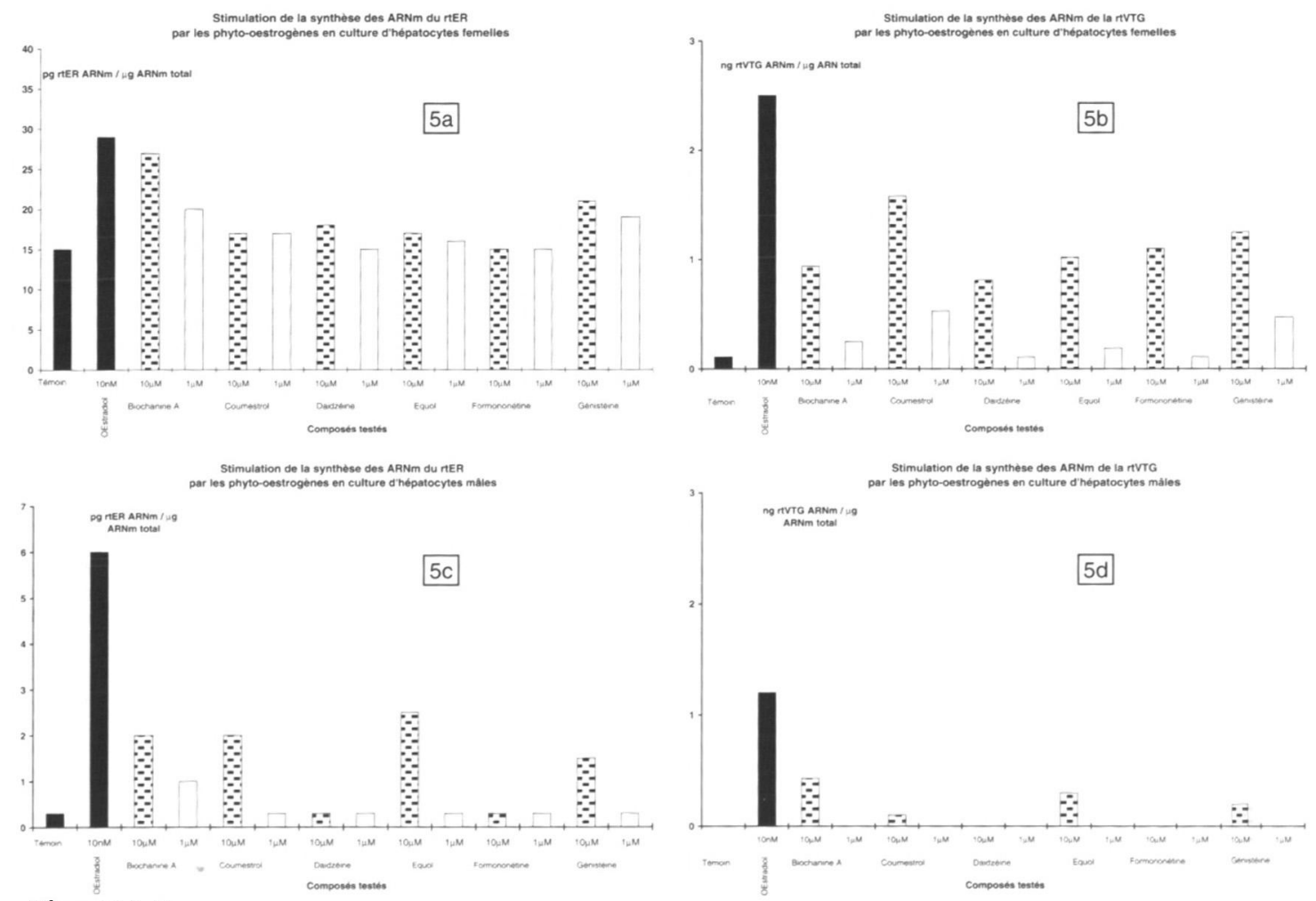

Figures 5

Effets in vitro des phyto-oestrogènes sur la synthèse des ARN messagers du récepteur de l'oestradiol et de la vitellogénine par des hépatocytes de truites femelles ( $5 a$ et $5 b$ respectivement) et de truites mâles ( $5 c$ et $5 d$ respectivement).

\section{Figure 5}

In vitro effects of phytoestrogens on estradiol receptor mRNA and vitellogenin mRNA in hepatocytes from female rainbow trout (5a and $5 \mathrm{~b}$ respectively) and from male rainbow trout ( $5 c$ and $5 d$ respectively). 
Cet élément est d'ailleurs confirmé par des données obtenues en mesurant les taux des ARN messagers du récepteur de l'oestradiol (rtER) et ceux des ARN messagers de la vitellogénine (rtVTG) sur des cultures d'hépatocytes obtenues à partir de foies prélevés chez des femelles ou des mâles (BENNETAU-PELISSERO et al., 1996).

Les Figures $5 a, 5 b, 5 c, 5 d$ montrent les effets des phyto-oestrogènes comparés à celui du témoin et l'effet induit par l'oestradiol. La Figure 5a montre les taux d'ARNm du rtER, $5 b$ ceux de la rtVTG dans les deux cas sur les cultures d'hépatocytes récupérés sur des femelles alors que $5 c$ et $5 d$ montrent respectivement les taux d'ARNm de rtER et d'ARNm de rtVTG obtenus sur des cellules issues de foies de mâles. Chez les femelles, comme en culture d'hépatocytes précédemment, on observe qu'il n'y a pas une grande variabilité dans la réponse des hépatocytes face aux différents composés. Chez les mâles, en revanche, les composés induisant les réponses les plus importantes sont ceux qui sont considérés comme les plus oestrogéniques classiquement et ceux qui nous avaient donné in vivo chez l'esturgeon les plus faibles réponses. On observe dans ces différents cas que l'induction des ARNm du récepteur de la vitellogénine est beaucoup plus faible dans le cas des mâles chez qui les taux de base des ARNm du récepteur de l'oestradiol sont faibles que chez les femelles chez qui les taux de base des ARNm du récepteur de l'oestradiol sont plus élevés.

On sait notamment que les protéines porteuses des stéroïdes peuvent jouer un rôle non négligeable dans le transfert des activités oestrogéniques (VERMEULEN 1986). Or, des travaux préliminaires semblent indiquer que seul l'équol déplace la liaison de la testostérone radioactive à la SBP de truite alors que chez l'esturgeon l'équol et la génistéine déplacent tous deux cette liaison (Figures $6 a$ et $b$ ).
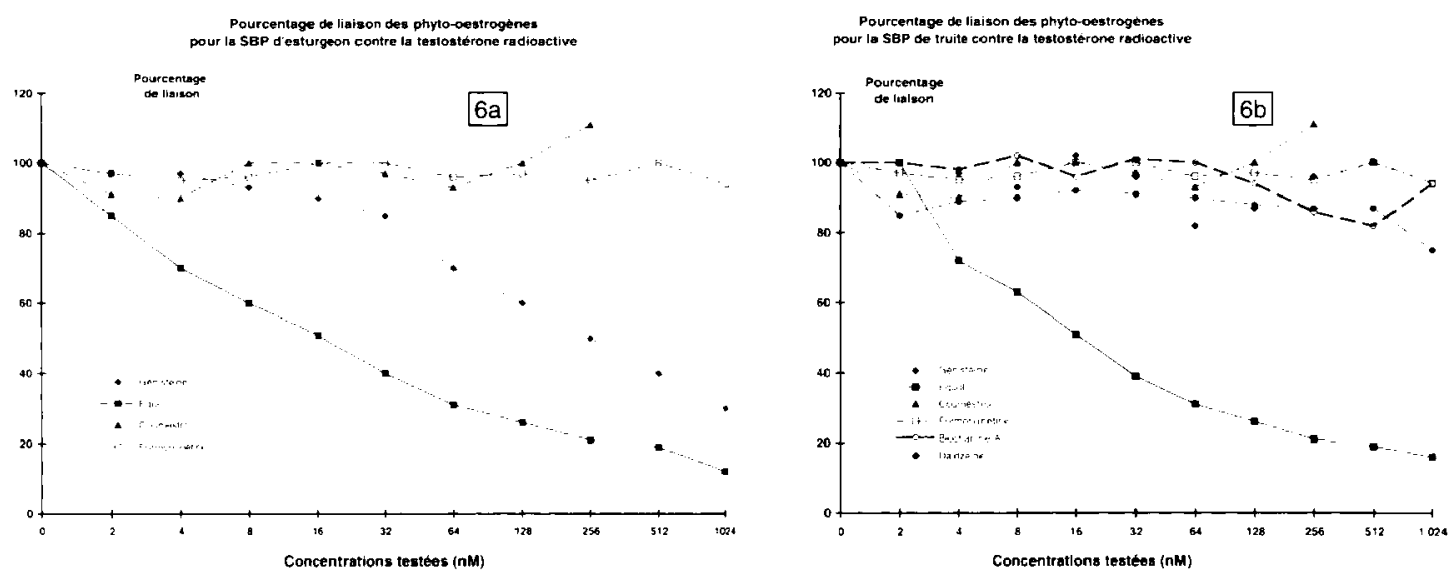

Figure 6a

La génistéine et l'équol sont tous deux capables de se lier à la SBP de l'esturgeon sibérien en déplaçant la liaison de la testostérone radioactive.

Figure 6a

Equol and genistein both bind to Siberian sturgeon SBP.

Figure 6b

Chez la truite arc-en-ciel, seul l'équol est capable de déplacer la liaison de la testostérone radioactive sur la SBP.

\section{Figure 6b}

In the rainbow trout, only equol binds to SBP. 
Enfin, il a été observé qu'un changement d'aliment se traduisait chez des femelles d'esturgeon en vitellogénèse par une diminution très importante des taux plasmatiques d'oestradiol et par une augmentation concomitante des taux de testostérone (PELISSERO et al., 1989a). Cette évolution très marquée suggère un blocage de l'aromatase et nous a incité à vérifier l'effet des phyto-oestrogènes in vitro sur l'aromatase ovarienne de la truite. Les résultats obtenus (PELISSERO et al., 1996) montrent là encore que seul l'équol est capable d'inhiber cette enzyme.

\section{DISCUSSION}

\section{Pourquoi l'esturgeon est-il plus sensible que la truite arc-en-ciel aux composés oestrogéniques du soja?}

Nous n'avons sans doute pas encore toutes les réponses à cette simple question mais nous possédons déjà un ensemble de résultats qui nous permettent d'apporter quelques éléments. Si l'on reprend point par point les éléments d'une action oestrogénique, on s'aperçoit qu'un certain nombre d'étapes sont nécessaires à la transmission de cette information. Pour être actif, des oestrogènes, stéroïdiens ou non, doivent :

1. être soit synthétisés de manière endogène, soit absorbés par voie digestive et/ou branchiale et conservés en l'état lors du passage dans le tractus intestinal ;

2. être transportés vers les cellules cibles par les protéines porteuses des stéroïdes ;

3. se lier aux récepteurs de l'oestradiol et se lier de manière suffisamment efficace pour induire la transcription des ARNm de la VTG ;

4. ne pas être éliminés trop rapidement par voie fécale ou urinaire sinon leur demivie dans le plasma étant très courte, ils n'ont pas le temps de se concentrer entre deux repas et d'interagir avec les cellules cibles ;

Si l'on reprend point par point ces différentes étapes, on remarque que :

1. la synthèse d'oestrogènes endogènes semble perturbée chez l'esturgeon par des composés d'origine alimentaire puisqu'un changement d'aliment se traduit par un effondrement des taux d'oestradiol et une augmentation concomitante des taux de testostérone (PELISSERO et al., 1996). Par ailleurs, les composés oestrogéniques du soja semblent subir chez l'esturgeon une transformation qui n'apparaît pas, semble-t-il, chez la truite. C'est la transformation de la génistéine en un composé plus polaire qui pourrait être un résidu dihydroxylé sur le cycle $b$. En revanche, chez la truite les études menées par CRAVEDI n'ont jamais fait apparaître ce composé notamment dans les sucs biliaires d'animaux ayant reçu une alimentation enrichie en génistéine. De plus, chez l'esturgeon, l'équol a été identifié dans le plasma des animaux par chromatographie en phase gazeuse couplée à la spectrométrie de masse (GCMS) (PELISSERO et al., 1989b). La présence de ce composé naturellement plus oestrogénique que la génistéine ainsi que celle du composé dihydroxylé sur le cycle b et dont l'activité oestrogénique est pour le moment inconnue pourrait en partie expliquer la plus grande sensibilité de l'esturgeon aux composés oestrogéniques du soja;

2. concernant le transport des oestrogéno-mimétiques du soja, on voit que là encore la truite et l'esturgeon diffèrent. Chez l'esturgeon, la génistéine et l'équol sont tout deux capables de déplacer la liaison de la testostérone à sa protéine porteuse. Or, d'après les données que nous possédons, ces deux composés sont présents dans le plasma des esturgeons lors de l'administration d'un régime à base de soja et de génistéine (CRAVEDI, 
communication personnelle) alors que chez la truite seule la génistéine est présente mais elle ne se lie pas aux SBP. Dans l'hypothèse où la SBP jouerait un rôle dans l'entrée des oestrogènes dans leurs cellules cibles, on voit que l'esturgeon a plus de chance de transmettre l'information oestrogénique des phyto-oestrogènes que la truite;

3. nous n'avons pas encore de réponse précise concernant des différences éventuelles dans le mécanisme d'action des oestrogènes du soja sur les cellules cibles de l'oestradiol. En effet, des cultures d'hépatocytes d'esturgeon n'ont jamais été réalisées et un blocage de l'action des phyto-oestrogènes par le tamoxiphène n'a jamais été testé. Il est possible que la spécificité du récepteur des oestrogènes chez l'esturgeon soit différente de celle de la truite et que ceci puisse avoir, là encore, une incidence sur la différence de réponse entre les deux espèces. Mais ceci reste du domaine de l'hypothèse ;

4. concernant le temps de résidence des phyto-oestrogènes dans le plasma des truites et des esturgeons, nous n'avons pas encore de données puisque nous n'avons pas encore la possibilité de doser ces composés dans le plasma des animaux. Toutefois, nous avons indirectement des éléments sur la durée de vie potentielle de ces composés chez les deux espèces en considérant l'activité de biotransformation des enzymes impliquées dans les mécanismes de détoxification des xénobiotiques. En effet, les phyto-oestrogènes peuvent être considérés comme des xénobiotiques qui, de part leurs structures et la forme sous laquelle ils se présentent dans les aliments, sont pris en charge par des enzymes impliqués dans l'élimination des stéroïdes. Notamment on sait, par des analyses chez d'autres vertébrés dont l'homme, que les phyto-oestrogènes sont éliminés essentiellement sous forme de gluco-conjugués et sulfo-conjugués dans l'urine et les fèces. Cette élimination implique l'action de l'uranyl diphosphate glucuronyl transférase (UDPGT) qui lie un résidu glucuronide sur les stéroïdes et participe ainsi à leur élimination par voie urinaire. II est intéressant de noter ici que PERDU-DURAND et CRAVEDI (1989) ont mis en évidence que cette enzyme était environ 10 fois moins active chez l'esturgeon que chez la truite. Ceci laisserait présager une élimination plus lente des phyto-oestrogènes par voie urinaire chez l'esturgeon, donc un temps de résidence plus long et donc une action plus importante.

\section{CONCLUSION}

II est probable, au vu des premiers résultats obtenus, que le soja et les composés oestrogéniques qu'il contient ne posent pas de problème majeur dans l'élevage de la truite arc-en-ciel. II reste toutefois à étayer cette théorie par des tests in vivo à partir de doses compatibles avec les concentrations rencontrées dans les aliments. En revanche, il semble qu'entre l'esturgeon hypersensible et la truite insensible il y ait la place pour d'autres espèces qui pourraient, au niveau d'une au moins des étapes du transfert de l'information oestrogénique, présenter une sensibilité supérieure à celle de la truite. II conviendrait sans doute d'explorer cette sensibilité sur d'autres espèces d'élevage surtout à l'heure où l'on parle de plus en plus de remplacer les sources de protéines animales par des sources de protéines végétales.

\section{BIBLIOGRAPHIE}

AXELSON M., SJÖVALL J., GUSTAFSSON B.E., SETCHELL K.D.R., 1982. Origin of lignans in mammals and identification of a precursor from plants. Nature, 298, 659-660.

BARNES S., 1995. Effect of genistein on in vitro and in vivo models of cancer. J. Nutr., $125,777 \mathrm{~S}-783 \mathrm{~S}$. 
BENNETAU-PELISSERO C., FLOURIOT G., VALOTAIRE Y., SUMPTER J.P., LE MENN F., 1996. Induction of rainbow trout estradiol receptor (rtER) MRNA and vitellogenin (tVTG) mRNA by phytoestrogens in hepatocyte cultures. 18th International Conference of Comparative Endocrinology, Rouen Septembre 1996. Annales d'endocrinologie, 57 (4), p. 30.

FARNWORTH N.R., BINGEL A.S., CORDELL G.A., CRANE F.A., FONG H.H.S., 1975. Potential value of plants as sources of new antifertility agents. I. J. Pharm. Sci., 64 (4), 535-598.

HESSELTINE C.W., ROGERS R.F., SHOTWELL O., 1978. Fungi, especially Gibberella zeae and zeralenone occurrence in wheat. Mycologia, 70, 14-18.

PELISSERO C., LE MENN F., WILLIOT P., 1989a. Evolution of sex steroid levels in male and first time maturing female of the Siberian sturgeon Acipenser baeri reared in fish farm. Proceed. First Int. Symp. Acipenser, Bordeaux 1989, 87-97.

PELISSERO C., LE MENN F., GARRIGUES P., 1989b. Detection of equol in the steroid fraction from sturgeon (Acipenser baeri) plasma using capillary gaz chromatography and mass spectrometry. The Analyst, 114, 1703-1705.

PELISSERO C., BENNETAU B., BABIN P., LE MENN F., DUNOGUĖS J., 1990a. The estrogenicity of certain isoflavones in the Siberian sturgeon Acipenser baeri. J. Steroid. Biochem. Molec. Biol., 38, 293-299.

PELISSERO C., LE MENN F., KAUSHIK S., 1990b. Estrogenic effect of dietary soja bean meal on vitellogenesis in cultured Siberian sturgeon Acipenser baeri. Gen. Comp. Endocrinol., 83, 447-457.

PELISSERO C., FOUCHER J.L., BENNETAU B., DUNOGUĖS J., FLOURIOT G., SUMPTER J., 1991. In vitro estrogenic activity of phytoestrogens on liver vitellogenin synthesis in the rainbow trout (Oncorhynchus mykiss). Proceed. Fourth Int. Symp. Reprod. Physiol. Fish., Norwich U.K., 1991, 247-249.

PELISSERO C., FLOURIOT G., FOUCHER J.L., BENNETAU B., DUNOGUĖS J., LE GAC F., SUMPTER J.P., 1993. Vitellogenin synthesis in hepatocyte culture, an in vitro test for the estrogenic potency of chemicals. J. Steroid. Biochem. Mol. Biol., 44, 263-272.

PELISSERO C., LENCZOWSKI M., CHINZI D., SUMPTER J.P., FOSTIER A., 1996. Effects of flavonoids on aromatase activity, an in vitro study. J. Steroid Biochem. Mol. Biol., 57 (3/4), 215-223.

PELISSERO-BENNETAU C., CORRAZE G., CRAVEDI J.P., HELOU C., DAVAIL-CUISSET B., LE MENN F., KAUSHIK S.J., 1997. Potential estrogenic effect of plant protein in rainbow trout. Thd. Int. Symp. Res. Aqua.,. Barcelone 24/08-27/08/97.

PERDU-DURAND E.F., CRAVEDI J.P., 1989. Characterization of xenobiotiques metabolising enzymes in sturgeon (Acipenser baeri). Comp. Biochem. Physiol., 93b 4, 921-928.

SETCHELL K.D.R., 1985. Naturally occuring non-steroidal estrogens of dietary origin. In Estrogens in the environment II., MCLACHLAN ed., Elsevier Publ. Inc., 69-85.

SHERWOOD R.T., OLAH A.F., OLESON W.H., JONES E.E., 1970. Effect of disease and injury on accumulation of flavonoid estrogen, coumestrol in alfalfa. Phytopathology, 54, 98-103.

STOB M., 1983. Naturally occuring food toxicants : estrogens. In Handbook of Naturally Occuring Food Toxicants, RECHCIGL M. Jr ed., CRC Press Inc., Boca Raton Florida, 81-100.

THOMPSON L.U., 1993. Potential health benefits and problems associated with antinutrients in foods. Food Res. Inter., 26, 131-149.

VERMEULEN A., 1986. TeBG and CBG as an index of endocrine function. Binding Proteins of Steroid hormones, Colloque INSERM 1986, 149, 383-396. 